\title{
Characteristics, Treatment, and Prognosis of Cracked Teeth: A Comparison with Data from 10 Years Ago
}

\author{
Tae Yeon Lee ${ }^{1}$ Sung Eun Yang ${ }^{2}$ Hye Min Kim² Min Joo Kye ${ }^{2}$ \\ ${ }^{1}$ Department of Conservative Dentistry, College of Medicine, \\ Yeouido ST. Mary's Hospital, The Catholic University of Korea, \\ Seoul, Republic of Korea \\ 2Department of Conservative Dentistry, College of Medicine, Seoul \\ St. Mary's Dental Hospital, The Catholic University of Korea, Seoul, \\ Republic of Korea
}

\begin{abstract}
Address for correspondence Sung Eun Yang, DDS, PhD, Department of Conservative Dentistry, College of Medicine, Seoul St. Mary's Dental Hospital, The Catholic University of Korea, Banpo-daero 222, Seocho-gu, Seoul 06591, Republic of Korea (e-mail: dentyeun@catholic.ac.kr).
\end{abstract}

Eur J Dent 2021;15:694-701.

\begin{abstract}
Keywords

- cracked teeth

- characteristics

- treatment

- prognosis

- probing depth

- symptoms

- apical lesion

Objectives The purpose of this study was to analyze and to compare retrospectively the characteristics, the treatment process, and the prognosis of cracked teeth by comparing recent data with data from 10 years ago.

Materials and Methods Sixty-eight cracked teeth from March 2009 to June 2010 (2009 data) and 185 cracked teeth from March 2019 to June 2020 (2019 data) were analyzed. The characteristics of cracked teeth and the treatment method depending on probing depth, caries, and symptoms, and prognosis depending on pocket depth and apical lesions were analyzed using $R$ version 3.3.3 (R Foundation for Statistical Computing, Vienna, Austria) and T\&F version 3.0 (Yoojin BioSoft, Korea). To compare proportions, the two-sample proportion test was performed. The distribution of proportions within the samples from 2009 and 2019 data was analyzed using the Chi-square test or binomial test. A $p$-value $<0.05$ was considered to indicate statistical significance.

Results Significantly fewer cracked teeth received root canal treatment before crown in 2019 than in 2009 ( $p=0.032)$. In both 2009 and 2019, symptomatic cracked teeth and those with deep periodontal pockets $(>6 \mathrm{~mm}$ ) were significantly more likely to receive root canal treatment. In both years, cracked teeth with a probing depth less than $6 \mathrm{~mm}$ or without an apical lesion were significantly more likely to be asymptomatic at 3-month and 6-month follow-ups $(p<0.001)$.

Conclusion Cracked teeth with a deep periodontal pocket or symptoms had a higher likelihood of endodontic treatment, and the presence of a deep periodontal pocket or apical lesion was associated with a higher risk of persistent symptoms. Therefore, clinicians should consider these factors when planning treatment and predicting patients' prognosis.
\end{abstract}

\section{Introduction}

Since cracked teeth syndrome was first described by Cameron, ${ }^{1}$ many authors have proposed different terms and

published online June 25, 2021
DOI https://doi.org/

$10.1055 / \mathrm{s}-0041-1728842$ ISSN 1305-7456 definitions for cracks in teeth. The American Association of Endodontics has identified five types of longitudinal tooth fractures in teeth, including craze line, fractured cusp, cracked tooth, split tooth, and vertical root fracture. ${ }^{2}$
(C) 2021. European Journal of Dentistry.

This is an open access article published by Thieme under the terms of the Creative Commons Attribution-NonDerivative-NonCommercial-License, permitting copying and reproduction so long as the original work is given appropriate credit. Contents may not be used for commercial purposes, or adapted, remixed, transformed or built upon. (https://creativecommons.org/licenses/by-nc-nd/4.0/).

Thieme Medical and Scientific Publishers Pvt. Ltd. A-12, 2nd Floor, Sector 2, Noida-201301 UP, India 
Cracked tooth is defined as an incomplete fracture that initiates from the crown and extends subgingivally, usually directed mesiodistally. ${ }^{2}$

The symptoms of cracked teeth can vary considerably depending on the length of the crack and the severity of pulp inflammation, ${ }^{3,4}$ which may occur as a result of microleakage-induced irritation and lead to thermal sensitivity. ${ }^{5,6}$ Although the most common symptom of cracked teeth is discomfort during chewing, sensitivity to cold with no other explanation also occurs frequently. ${ }^{7}$ Because it is difficult to locate crack lines and cracked teeth have complex and wide-ranging symptoms, this condition is difficult to diagnose in clinical trials.

Moreover, predicting the prognosis of a cracked tooth is difficult due to the lack of a definitive method of establishing how far the crack has progressed. ${ }^{8,9}$ It was reported that the likelihood of success decreased as pulpal involvement increased (85\% for teeth with irreversible pulpitis, $80 \%$ for necrotic teeth, and $74 \%$ for previously treated teeth). ${ }^{5,9,10}$ The difficulty of predicting the prognosis of cracked teeth, including whether extraction will be necessary, poses a dilemma for clinicians. ${ }^{5,11}$ Thus, there is a need to identify cracked teeth-related factors that may provide useful information for treatment planning and predicting patients' prognosis. This study compared data over the past 10 years to analyze changes in the treatment method of cracked teeth and to reconfirm the factors that influence the prognosis. Specifically, this study analyzed the characteristics, treatment process, and prognosis of cracked teeth based on a comparison of recent data with data from 10 years ago.

\section{Materials and Methods}

\section{Ethical Considerations}

The protocol for this study was approved by the Institutional Review Board of Seoul St. Mary's Dental Hospital, The Catholic University of Korea, Seoul, Korea (KC20RISI0187).

\section{Data Collection Procedure}

Records on 476 crown-restored teeth from March 2009 to June 2010 (2009 data) and 1,913 crown-restored teeth from March 2019 to June 2020 (2019 data) from the Department of Conservative Dentistry at Seoul St Mary's Dental Hospital were screened and evaluated. Dental records were reviewed by two endodontists, and the following inclusion and exclusion criteria were applied:

1. Patient whose diagnosis were recorded as cracked teeth were selected.

2. It was confirmed that the diagnostic procedure included a bite test and crack line visualization.

3. Teeth with a craze line, cusp fracture, or split were excluded.

4. Teeth with incomplete clinical records were excluded.

A retrospective study was conducted of 68 cracked teeth from 2009 and 185 from 2019. At the 3-month follow-up time point, 45 teeth from 2009 and 180 teeth from 2019 were analyzed, and the 6-month follow-up data included 29 teeth treated in 2009 and 50 teeth treated in 2019. The decrease in the number of teeth at each follow-up time point occurred because some patients were lost to follow-up.

The cracked teeth were diagnosed by a bite test using a cotton roll and wood stick, and the periodontal probing depth corresponding to the crack was analyzed. Examination by the naked eye, transillumination, staining with methylene blue dye, and microscopy were also performed to diagnose the crack. Tooth hypersensitivity was determined using an ice stick and a thermal pulp tester (Frigi-Dent; Ellman international Inc., Oceanside, New York, United States). If it showed no response, we verified it again using an electric pulp tester (Digitest; Parkell Inc., Farmingdale, New York, United States). Periapical radiographs were taken to confirm apical changes.

Diagnoses were verified during the treatment process. General and pretreatment data of the cracked teeth were collected from patients' clinical records. Age and sex, the location of the tooth, the presence of a restoration, restoration type and the filling material, the presence of cervical abrasion, the presence of caries, number of crack lines, ice test results, bite test results, percussion test results, and periodontal pocket depth were noted. If patients showed bite pain or cold sensitivity, cracked teeth were classified as symptomatic.

Cracked teeth were managed following the treatment protocol shown in - Fig. 1. If a cracked tooth was diagnosed as symptomatic irreversible pulpitis or as pulp necrosis, root canal treatment was performed.

At 3- and 6-month follow-up examinations, signs and symptoms were recorded, including bite pain, percussion, cold sensitivity, sinus tract, swelling, and periodontal pocket associated with the crack. A periodontal pocket was defined as a periodontal probing depth over $4 \mathrm{~mm}$.

Cracked teeth-related factors were analyzed and compared between the samples of 2009 and 2019. Furthermore, comparative analyses were conducted of treatment procedures according to pocket depth and presence or absence of caries and symptoms and of prognosis according to pocket depth and presence or absence of an apical lesion in the samples of 2009 and 2019.

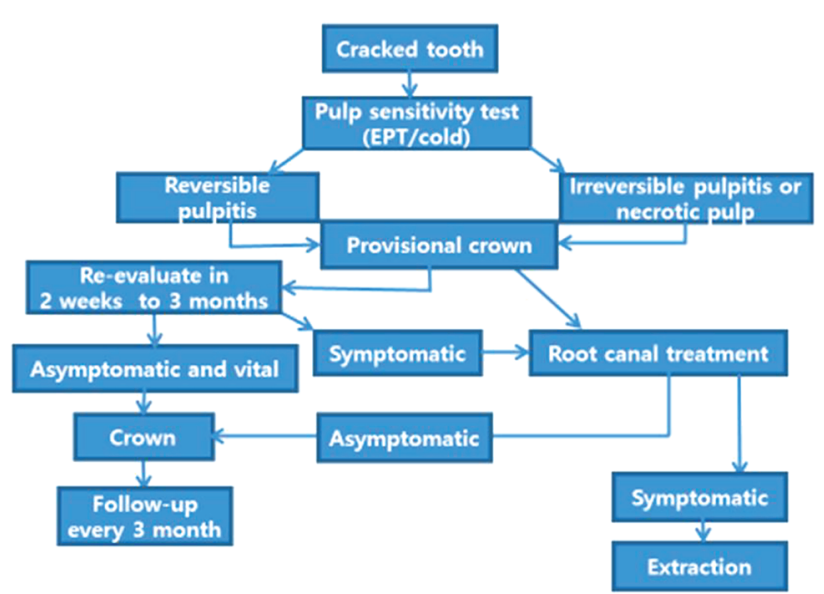

Fig. 1 The different treatment protocols according to pulpal and periapical diagnosis of cracked teeth. 


\section{Statistical Analysis}

The results were analyzed using R version 3.3.3 (R Foundation for Statistical Computing, Vienna, Austria) and T\&F version 3.0 (Yoojin BioSoft, Korea). Data were expressed as number and percentage. To compare proportions in the samples of 2009 and 2019, the two-sample proportion test was performed. The distribution of proportions within the samples from 2009 and 2019 data was analyzed using the Chi-square test or binomial test. A $p$-value $<0.05$ was considered to indicate statistical significance.

\section{Results}

This study analyzed 68 cracked teeth from 2009 and 185 from 2019. The incidence rate of cracked teeth was $14.3 \%$ (68/476) in 2009 and $9.7 \%(185 / 1913)$ in 2019. The incidence rate in 2009 was significantly higher than in 2019 ( $p=0.004$; two-sample proportion test).

Factors associated with cracked teeth are presented in - Table 1. The mandibular first molars were most commonly involved (33.8\%) in 2009, and the mandibular second molars (28.1\%) were most commonly involved in 2019. The incidence rate of cracked teeth in mandibular second molars in 2019 was significantly higher than in 2009. Cracked teeth exhibited a single crack line more frequently in 2019 (70.8\%) than in $2009(p<0.001)$.

Cracked teeth were mainly treated by a permanent crown after a provisional crown (prov $\rightarrow \mathrm{Cr}$ ), a permanent crown after root canal treatment and provisional crown (RCT and prov $\rightarrow \mathrm{Cr}$ ), and root canal treatment after a permanent crown (prov $\rightarrow \mathrm{Cr} \rightarrow \mathrm{RCT}$ ). The distribution is presented in - Table 2 . In 2009, cracked teeth were mainly treated with RCT and prov $\rightarrow \operatorname{Cr}(52.9 \%)$. In 2019, cracked teeth were mainly treated with prov $\rightarrow \mathrm{Cr}$ (55.4\%). The proportion of teeth treated with RCT and prov $\rightarrow \mathrm{Cr}$ showed a significant decrease $(p=0.032)$ in 2019 compared with 2009.

The treatment protocols for cracked teeth according to periodontal probing depth in 2009 and 2019 are presented in - Table 3. In both 2009 and 2019, a deeper periodontal probing depth was associated with a higher likelihood of root canal treatment and a lower likelihood of treatment with prov $\rightarrow \mathrm{Cr}$, but there was no significant difference in the treatment method.

- Table 4 presents the treatment protocols for cracked teeth depending on the presence or absence of symptoms and caries in 2009 and 2019. In both years, symptomatic cracked teeth were significantly more likely to receive root canal treatment than asymptomatic cracked teeth $(75 \%, p=$ 0.005 in 2009; 61.8\%, $p$ < 0.001 in 2019). In both 2009 and 2019, asymptomatic cracked teeth were significantly more likely to receive prov $\rightarrow \mathrm{Cr}$ than symptomatic cracked teeth (55\%, $p=0.027$ in 2009; 75.9\%, $p<0.001$ in 2019).

The prognosis of cracked teeth according to the periodontal probing depth at 3 and 6 months after treatment in 2009 and 2019 is presented in -Table 5. At 3 and 6 months of follow-up, both in 2009 and 2019, teeth with a deep periodontal pocket $(>6 \mathrm{~mm}$ ) did not show a significant difference in terms of whether symptoms disappeared. In contrast, symptoms were significantly more likely to disappear in teeth with a mild or moderate periodontal probing depth $(<3 \mathrm{~mm}$, 4-6 mm). At 6-month follow-up, in 2019, the proportion of teeth with a deep periodontal pocket $(>6 \mathrm{~mm})$ in which symptoms disappeared was higher than that at the 3-month follow-up (71.4\% at 3 months, $85.7 \%$ at 6 months).

The prognosis of cracked teeth depending on the presence of an apical lesion at 3 and 6 months after treatment in 2009 and 2019 is presented in - Table 6. At both time points in both years, teeth without an apical lesion in the initial examination were significantly more likely to show symptom disappearance than symptom persistence $(p<0.001)$.

The survival rate of the teeth from 2019 at the 1-year follow-up was $100 \%$. Of the teeth treated in 2009 , three teeth were extracted (at 2 years and 1 month, 2 years and 10 months, and 10 years and 5 months), corresponding to a survival rate of $95 \%$.

\section{Discussion}

In this study, changes in the characteristics, treatment methods, and prognosis of cracked teeth were analyzed by comparing data of 2009 and 2019. Several studies have been conducted on cracked teeth, but this is the first study to compare data of 10 years ago to recent data.

The incidence of cracked teeth was lower in 2019 (9.7\%) than in 2009 (14.3\%). A recent study reported an incidence rate of $8.9 \%$, similar to that in $2019 .{ }^{2}$ One reason for this difference may be that the study in 2019 was conducted with a larger sample. Another reason may be that the referral rate of cracked teeth to tertiary medical institutions was lower in 2019 than in 2009 because of improvements in general dentists' understanding of cracked teeth over the course of this 10-year period. Generally, the characteristics of cracked teeth in this study were not significantly different from those previously reported in the literature. In both years, similar results were found for factors related to age, sex, and the presence of an old restoration, cervical abrasion, or caries, while different results were found regarding the location of cracked teeth and crack lines.

In 2019, the incidence rate of cracked tooth was significantly higher in mandibular second molars than it was in 2009. Cracked teeth have been reported to be most prevalent in mandibular molars, ${ }^{1,4,12-14}$ maxillary premolars, ${ }^{7,15}$ maxillary molars, ${ }^{16}$ and maxillary first molars. ${ }^{14,17}$ In recent studies, ${ }^{12,13,18}$ cracked teeth were reported to be the most prevalent in the mandibular second molars. This tendency may be due to the location of these teeth near the temporomandibular joint, ${ }^{19}$ where the lever effect may cause relatively high masticatory forces to be exerted on teeth. The lingual cusps of maxillary molars have been suggested to play the role of plungers, causing structural fatigue in the lower antagonists. ${ }^{20,21}$

In 2019, the prevalence of cracked teeth with a single crack line was significantly higher than that in 2009 , similar to the results of recent studies. ${ }^{14}$ The results regarding the characteristics of cracked teeth in 2019 were more similar 
Table 1 Associations between various factors and cracked teeth in 2009 and 2019

\begin{tabular}{|c|c|c|c|c|c|c|}
\hline Variable & Subgroup & $\begin{array}{l}\text { Year: } 2009 \\
(N=68)\end{array}$ & $p$-Value (1) & $\begin{array}{l}\text { Year: } 2019 \\
(N=185)\end{array}$ & $p$-Value (2) & $p$-Value (3) \\
\hline Sample no. & & $N=68$ & & $N=185$ & & \\
\hline \multirow[t]{9}{*}{ Location of tooth } & & & $<0.001$ & & $<0.001$ & \\
\hline & Mandibular first molar & $23(33.8)$ & & $42(22.7)$ & & 0.103 \\
\hline & Mandibular first premolar & $2(2.9)$ & & $2(1.1)$ & & 0.629 \\
\hline & Mandibular second molar & $5(7.4)$ & & $52(28.1)$ & & $<0.001$ \\
\hline & Mandibular second premolar & $1(1.5)$ & & $5(2.7)$ & & 0.916 \\
\hline & Maxillary first molar & $12(17.6)$ & & $38(20.5)$ & & 0.738 \\
\hline & Maxillary first premolar & $10(14.7)$ & & $6(3.2)$ & & 0.002 \\
\hline & Maxillary second molar & $7(10.3)$ & & $31(16.8)$ & & 0.281 \\
\hline & Maxillary second premolar & $8(11.8)$ & & $9(4.9)$ & & 0.097 \\
\hline \multirow[t]{7}{*}{ Age, years } & & & $<0.001$ & & $<0.001$ & \\
\hline & $20-29$ & $12(17.6)$ & & $4(2.2)$ & & $<0.001$ \\
\hline & $30-39$ & $5(7.4)$ & & $27(14.6)$ & & 0.186 \\
\hline & $40-49$ & $11(16.2)$ & & $40(21.6)$ & & 0.435 \\
\hline & $50-59$ & $21(30.9)$ & & $51(27.6)$ & & 0.718 \\
\hline & $60-69$ & $18(26.5)$ & & $41(22.2)$ & & 0.582 \\
\hline & $\geq 70$ & $1(1.5)$ & & $22(11.9)$ & & 0.021 \\
\hline \multirow[t]{3}{*}{ Sex } & & & 0.628 & & 0.023 & \\
\hline & $\mathrm{F}$ & $36(52.9)$ & & $108(58.4)$ & & 0.528 \\
\hline & M & $32(47.1)$ & & 77 (41.6) & & 0.528 \\
\hline \multirow[t]{11}{*}{ Type of restorative material } & & & $<0.001$ & & $<0.001$ & \\
\hline & $\mathrm{Cl} 1$ Amalgam. & $8(11.8)$ & & $19(10.3)$ & & 0.911 \\
\hline & Cl 1 Ceramic inlay & $0(0)$ & & $3(1.6)$ & & 0.688 \\
\hline & Cl 1 Gold inlay & $11(16.2)$ & & $44(23.8)$ & & 0.259 \\
\hline & Cl 1 Resin & $4(5.9)$ & & $16(8.6)$ & & 0.645 \\
\hline & $\mathrm{Cl} 2$ Amalgam. & $4(5.9)$ & & $2(1.1)$ & & 0.079 \\
\hline & $\mathrm{Cl} 2$ Ceramic inlay & $0(0)$ & & $2(1.1)$ & & 0.952 \\
\hline & $\mathrm{Cl} 2$ Gold inlay & $3(4.4)$ & & $15(8.1)$ & & 0.460 \\
\hline & Crown & $2(2.9)$ & & $11(5.9)$ & & 0.523 \\
\hline & No restoration & $35(51.5)$ & & $68(36.8)$ & & 0.049 \\
\hline & Temporary filling & $1(1.5)$ & & $5(2.7)$ & & 0.916 \\
\hline \multirow[t]{3}{*}{ Cervical abrasion } & & & $<0.001$ & & $<0.001$ & \\
\hline & Present & $3(4.4)$ & & $15(8.1)$ & & 0.460 \\
\hline & Absent & 65 (95.6) & & 170 (91.9) & & 0.460 \\
\hline \multirow[t]{3}{*}{ Caries } & & & 0.015 & & $<0.001$ & \\
\hline & Present & $24(35.3)$ & & $42(22.7)$ & & 0.063 \\
\hline & Absent & $44(64.7)$ & & $143(77.3)$ & & 0.063 \\
\hline \multirow[t]{3}{*}{ Crack line } & & & 0.002 & & $<0.001$ & \\
\hline & 1 & $21(30.9)$ & & $131(70.8)$ & & $<0.001$ \\
\hline & $>2$ & $47(69.1)$ & & $54(29.2)$ & & $<0.001$ \\
\hline
\end{tabular}

Note: Data were expressed as number and percentage. Distribution of sample ratios among each subgroup within the data from 2009 or 2019 was analyzed using the Chi-square test, yielding $p$-value (1) and $p$-value (2). To compare sample ratios between the data from 2009 and 2019 , the two-sample proportion test was performed, yielding $p$-value (3). A $p$-value $<0.05$ was considered to indicate statistical significance. 
Table 2 Distribution of treatment strategies for managing cracked teeth

\begin{tabular}{|l|l|l|l|l|l|l|l|}
\hline & 2009 & $N=68$ & 2019 & $N=184$ & $p$-Value \\
\cline { 2 - 8 } & $\mathrm{F}$ & $\mathrm{M}$ & Total $N(\%)$ & $\mathrm{F}$ & $\mathrm{M}$ & Total $N(\%)$ & \\
\hline Prov $\rightarrow \mathrm{Cr}$ & 16 & 13 & $29(42.6)$ & 62 & 40 & $102(55.4)$ & 0.097 \\
\hline Prov $\rightarrow \mathrm{Cr} \rightarrow \mathrm{RCT}$ & 2 & 1 & $3(4.4)$ & 6 & 8 & $14(7.6)$ & 0.538 \\
\hline Prov and $\mathrm{RCT} \rightarrow \mathrm{Cr}$ & 18 & 18 & $36(52.9)$ & 39 & 29 & $68(37)$ & 0.032 \\
\hline
\end{tabular}

Abbreviations: $\mathrm{Cr}$, permanent crown; Prov, provisional crown; RCT, root canal treatment.

Note: To compare total sample ratios between the data of 2009 and 2019, the two-sample proportion test was performed. A $p$-value $<0.05$ was considered to indicate statistical significance.

Table 3 Different treatment protocols for cracked teeth according to the periodontal probing depth

\begin{tabular}{|c|c|c|c|c|c|}
\hline Year & PPD & Total $N$ & Prov $\rightarrow \mathrm{Cr}$ & Prov $\rightarrow \mathrm{Cr} \rightarrow \mathrm{RCT}$ & Prov and $\mathrm{RCT} \rightarrow \mathrm{Cr}$ \\
\hline \multirow[t]{7}{*}{2009} & $<3 \mathrm{~mm}$ & $40(100)$ & $20(50)$ & $3(7.5)$ & $17(42.5)$ \\
\hline & $4-6 \mathrm{~mm}$ & $25(100)$ & $9(36)$ & $0(0)$ & $16(64)$ \\
\hline & $>6 \mathrm{~mm}$ & $3(100)$ & $0(0)$ & $0(0)$ & $3(100)$ \\
\hline & Total & $68(100)$ & $29(42.6)$ & $3(4.4)$ & 36 (52.9) \\
\hline & $p$-Value [1] & & 0.396 & 0.427 & 0.152 \\
\hline & $p$-Value [2] & & 0.283 & 1.000 & 0.185 \\
\hline & $p$-Value [3] & & 0.544 & - & 0.544 \\
\hline \multirow[t]{7}{*}{2019} & $<3 \mathrm{~mm}$ & $117(100)$ & $68(58.1)$ & $10(8.5)$ & $39(33.3)$ \\
\hline & $4-6 \mathrm{~mm}$ & $53(100)$ & $28(52.8)$ & $4(7.5)$ & 21 (39.6) \\
\hline & $>6 \mathrm{~mm}$ & $14(100)$ & $6(42.9)$ & $0(0)$ & $8(57.1)$ \\
\hline & Total & $184(100)$ & $102(55.4)$ & $14(7.6)$ & $68(37)$ \\
\hline & $p$-Value [1] & & 0.633 & 1.000 & 0.534 \\
\hline & $p$-Value [2] & & 0.422 & 0.545 & 0.144 \\
\hline & $p$-Value [3] & & 0.716 & 0.670 & 0.382 \\
\hline \multirow[t]{7}{*}{$2009+2019$} & $<3 \mathrm{~mm}$ & $157(100)$ & $88(56.1)$ & $13(8.3)$ & $56(35.7)$ \\
\hline & $4-6 \mathrm{~mm}$ & $78(100)$ & $37(47.4)$ & $4(5.1)$ & $37(47.4)$ \\
\hline & $>6 \mathrm{~mm}$ & $17(100)$ & $6(35.3)$ & $0(0)$ & $11(64.7)$ \\
\hline & Total & $252(100)$ & $131(52)$ & $17(6.7)$ & $104(41.3)$ \\
\hline & $p$-Value [1] & & 0.268 & 0.541 & 0.111 \\
\hline & $p$-Value [2] & & 0.169 & 0.455 & 0.038 \\
\hline & $p$-Value [3] & & 0.521 & 0.774 & 0.306 \\
\hline
\end{tabular}

Abbreviations: $\mathrm{Cr}$, permanent crown; PPD, periodontal probing depth; Prov, provisional crown; RCT, root canal treatment.

Note: $p$-Values were computed using the two-sample proportion test to analyze differences in sample proportions among the PPD subgroups. $p$-Value [1]: between $<3$ and 4-6 mm. $p$-Value [2]: between $<3$ and $>6 \mathrm{~mm}$. $p$-Value [3]: between 4-6 and $>6 \mathrm{~mm}$. A $p$-value $<0.05$ was considered to indicate statistical significance.

to those of recent studies than the results from 2009. This is thought to be due to an increase in the number of patients and an increase in the accuracy of diagnoses of cracked teeth in 2019.

A prior study found cracks to occur more frequently in heavily restored teeth. ${ }^{7}$ However, recent studies have found a high incidence of cracks in unrestored teeth or teeth with class I restorations. ${ }^{12,14,16}$ Similarly, in this study, the highest proportion of cracks (51.5\% in 2009, 36.8\% in 2019) was found in intact teeth or in teeth with class I restorations.

Cracked teeth occurred more often in intact teeth than in carious teeth (95.6\% in 2009, 91.9\% in 2019). According to previous research, the weakening of tooth structures due to large restorations does not increase the incidence of cracked teeth; instead, cracks in intact teeth progress due to internal structural weaknesses present where masticatory forces are exerted upon calcification sites, with additional contributions made by parafunctional habits. ${ }^{15,19,20,22}$ Furthermore, it can be concluded that caries-induced weakening of tooth structures does not increase the incidence of cracked teeth.

In cracked teeth, loss of pulp vitality may predict an unfavorable prognosis, as the 2-year survival rate of cracked teeth that received endodontic treatment was reported to be only $85.5 \% .^{9}$ Therefore, detecting cracked teeth early and reducing the likelihood of root canal treatment can increase the long-term success rate. When comparing the treatment strategies used for cracked teeth in 2009 and 2019, RCT and 
Characteristics, Treatment, and Prognosis of Cracked Teeth Lee et al. 699

Table 4 Different treatment protocols for cracked teeth according to the presence of symptoms or caries

\begin{tabular}{|c|c|c|c|c|c|c|c|c|c|}
\hline \multirow[t]{2}{*}{ Year } & & Caries & & & & Symptoms & & & \\
\hline & Caries & Total $N$ & Prov $\rightarrow \mathrm{Cr}$ & $\begin{array}{l}\text { Prov } \rightarrow \\
\mathrm{Cr} \rightarrow \mathrm{RCT}\end{array}$ & $\begin{array}{l}\mathrm{RCT} \text { and } \\
\text { prov } \rightarrow \mathrm{Cr}\end{array}$ & Total $N$ & Prov $\rightarrow \mathrm{Cr}$ & $\begin{array}{l}\text { Prov } \rightarrow \\
\mathrm{Cr} \rightarrow \mathrm{RCT}\end{array}$ & $\begin{array}{l}\mathrm{RCT} \text { and } \\
\text { prov } \rightarrow \mathrm{Cr}\end{array}$ \\
\hline \multirow[t]{4}{*}{2009} & $\mathrm{O}$ & 24 & $10(41.7)$ & $0(0)$ & $14(58.3)$ & 28 & $7(25)$ & $0(0)$ & $21(75)$ \\
\hline & $x$ & 44 & $19(43.2)$ & $3(6.8)$ & $22(50)$ & 40 & $22(55)$ & $3(7.5)$ & $15(37.5)$ \\
\hline & Total & 68 & $29(42.6)$ & $3(4.4)$ & $36(52.9)$ & 68 & $29(42.6)$ & $3(4.4)$ & 36 (52.9) \\
\hline & $p$-Value & & 1.000 & 0.490 & 0.686 & & 0.027 & 0.378 & 0.005 \\
\hline \multirow[t]{4}{*}{2019} & $\mathrm{O}$ & 41 & $24(58.5)$ & $3(7.3)$ & $14(34.1)$ & 76 & $20(26.3)$ & $9(11.8)$ & $47(61.8)$ \\
\hline & $X$ & 143 & $78(54.5)$ & $11(7.7)$ & $54(37.8)$ & 108 & $82(75.9)$ & $5(4.6)$ & 21 (19.4) \\
\hline & Total & 184 & $102(55.4)$ & $14(7.6)$ & $68(37)$ & 184 & $102(55.4)$ & $14(7.6)$ & $68(37)$ \\
\hline & $p$-Value & & 0.783 & 1.000 & 0.811 & & $<0.001$ & 0.125 & $<0.001$ \\
\hline \multirow[t]{4}{*}{$2009+2019$} & $\mathrm{O}$ & 65 & $34(52.3)$ & $3(4.6)$ & $28(43.1)$ & 104 & $27(26)$ & $9(8.7)$ & $68(65.4)$ \\
\hline & $x$ & 187 & 97 (51.9) & $14(7.5)$ & $76(40.6)$ & 148 & $104(70.3)$ & $8(5.4)$ & $36(24.3)$ \\
\hline & Total & 252 & $131(52)$ & $17(6.7)$ & $104(41.3)$ & 252 & $131(52)$ & $17(6.7)$ & $104(41.3)$ \\
\hline & $p$-Value & & 1.000 & 0.611 & 0.844 & & $<0.001$ & 0.449 & $<0.001$ \\
\hline
\end{tabular}

Abbreviations: $\mathrm{Cr}$, permanent crown; Prov, provisional crown; RCT, root canal treatment.

Note: $p$-Values were computed to compare sample ratios between $\mathrm{O}$ and $\mathrm{X}$ using the two-sample proportion test. A $p$-value $<0.05$ was considered to indicate statistical significance.

Table 5 Prognosis of cracked teeth according to the probing depth at 3 and 6 mo after treatment

\begin{tabular}{|l|l|l|l|l|l|l|l|l|l|l|l|l|}
\hline & \multicolumn{9}{|c|}{3 mo } & \multicolumn{7}{|c|}{} & \multicolumn{5}{|c|}{6 mo } \\
\hline Year & PPD & Total $N$ & Free & Persisted & Ext & $p$-Value & Total $N$ & Free & Persisted & Ext & $p$-Value \\
\hline 2009 & $<3$ & 26 & $22(84.6)$ & $4(15.4)$ & $0(0)$ & $<0.001$ & 16 & $14(87.5)$ & $2(12.5)$ & $0(0)$ & 0.004 \\
\hline & $4-6$ & 16 & $15(93.8)$ & $1(6.2)$ & $0(0)$ & $<0.001$ & 11 & $11(100)$ & $0(0)$ & $0(0)$ & $<0.001$ \\
\hline & $>6$ & 3 & $0(0)$ & $3(100)$ & $0(0)$ & 0.250 & 2 & $0(0)$ & $2(100)$ & $0(0)$ & 0.500 \\
\hline & Total & 45 & $37(82.2)$ & $8(17.8)$ & $0(0)$ & $<0.001$ & 29 & $25(86.2)$ & $4(13.8)$ & $0(0)$ & $<0.001$ \\
\hline & $<3$ & 113 & $110(97.3)$ & $3(2.7)$ & $0(0)$ & $<0.001$ & 28 & $25(89.3)$ & $3(10.7)$ & $0(0)$ & $<0.001$ \\
\hline & $4-6$ & 53 & $41(77.4)$ & $12(22.6)$ & $0(0)$ & $<0.001$ & 15 & $12(80)$ & $3(20)$ & $0(0)$ & 0.035 \\
\hline & $>6$ & 14 & $10(71.4)$ & $4(28.6)$ & $0(0)$ & 0.180 & 7 & $6(85.7)$ & $1(14.3)$ & $0(0)$ & 0.125 \\
\hline & Total & 180 & $161(89.4)$ & $19(10.6)$ & $0(0)$ & $<0.001$ & 50 & $43(86)$ & $7(14)$ & $0(0)$ & $<0.001$ \\
\hline & $<3$ & 139 & $132(95)$ & $7(5)$ & $0(0)$ & $<0.001$ & 44 & $39(88.6)$ & $5(11.4)$ & $0(0)$ & $<0.001$ \\
\hline & $4-6$ & 69 & $56(81.2)$ & $13(18.8)$ & $0(0)$ & $<0.001$ & 26 & $23(88.5)$ & $3(11.5)$ & $0(0)$ & $<0.001$ \\
\hline & $>6$ & 17 & $10(58.8)$ & $7(41.2)$ & $0(0)$ & 0.629 & 9 & $6(66.7)$ & $3(33.3)$ & $0(0)$ & 0.508 \\
\hline & Total & 225 & $198(88)$ & $27(12)$ & $0(0)$ & $<0.001$ & 79 & $68(86.1)$ & $11(13.9)$ & $0(0)$ & $<0.001$ \\
\hline
\end{tabular}

Abbreviation: PPD, periodontal probing depth.

Note: $p$-Values were computed to compare sample ratio difference between free and persisted according to PPD using the binomial test. A $p$-value $<0.05$ was considered to indicate statistical significance.

prov $\rightarrow$ Cr was significantly less common in 2019 (37\%) than in 2009 (52.9\%). The lower frequency of root canal treatment in 2019 was likely due to an increase in early detection of cracked teeth, which resulted from an improved understanding of the characteristics of cracked teeth and the more precise diagnosis of cracked teeth.

In this study, we investigated whether the rate of root canal treatment was higher in cracked teeth with caries.
In both 2009 and 2019, no correlation was observed between the presence of caries in the initial examination and the treatment method of cracked teeth.

The most common symptom of cracked teeth is chewing-associated discomfort; however, sensitivity to cold without an alternative explanation is also a frequent symptom. ${ }^{7}$ The location and extent of the crack, as well as the presence of periapical disease and the condition of the 
Table 6 Prognosis of cracked teeth according to the presence of an apical lesion at 3 and 6 mo after treatment

\begin{tabular}{|c|c|c|c|c|c|c|c|c|c|c|c|}
\hline \multicolumn{7}{|l|}{$3 \mathrm{mo}$} & \multicolumn{5}{|l|}{$6 \mathrm{mo}$} \\
\hline Year & $\begin{array}{l}\text { Apical } \\
\text { lesion }\end{array}$ & Total $N$ & Free & Persisted & Ext & $p$-Value & Total $N$ & Free & Persisted & Ext & $p$-Value \\
\hline \multirow[t]{3}{*}{2009} & $\mathrm{O}$ & 6 & $5(83.3)$ & 1 (16.7) & $0(0)$ & 0.219 & 4 & $4(100)$ & $0(0)$ & $0(0)$ & 0.125 \\
\hline & $x$ & 39 & $32(82.1)$ & 7 (17.9) & $0(0)$ & $<0.001$ & 25 & $21(84)$ & $4(16)$ & $0(0)$ & $<0.001$ \\
\hline & Total & 45 & $37(82.2)$ & $8(17.8)$ & $0(0)$ & $<0.001$ & 29 & $25(86.2)$ & $4(13.8)$ & $0(0)$ & $<0.001$ \\
\hline \multirow[t]{3}{*}{2019} & $\mathrm{O}$ & 25 & $19(76)$ & $6(24)$ & $0(0)$ & 0.015 & 11 & $9(81.8)$ & $2(18.2)$ & $0(0)$ & 0.065 \\
\hline & $X$ & 155 & 142 (91.6) & $13(8.4)$ & $0(0)$ & $<0.001$ & 39 & $34(87.2)$ & $5(12.8)$ & $0(0)$ & $<0.001$ \\
\hline & Total & 180 & $161(89.4)$ & 19 (10.6) & $0(0)$ & $<0.001$ & 50 & $43(86)$ & $7(14)$ & $0(0)$ & $<0.001$ \\
\hline \multirow[t]{3}{*}{$2009+2019$} & $\mathrm{O}$ & 31 & $24(77.4)$ & 7 (22.6) & $0(0)$ & 0.003 & 15 & $13(86.7)$ & $2(13.3)$ & $0(0)$ & 0.007 \\
\hline & $x$ & 194 & $174(89.7)$ & $20(10.3)$ & $0(0)$ & $<0.001$ & 64 & $55(85.9)$ & $9(14.1)$ & $0(0)$ & $<0.001$ \\
\hline & Total & 225 & $198(88)$ & $27(12)$ & $0(0)$ & $<0.001$ & 79 & $68(86.1)$ & $11(13.9)$ & $0(0)$ & $<0.001$ \\
\hline
\end{tabular}

Note: $p$-Values were computed to compare sample ratio difference between free and persisted according to apical lesion using the binomial test. A $p$-value $<0.05$ was considered to indicate statistical significance.

pulp, affect the symptoms of cracked teeth. ${ }^{18,23}$ In this study, we examined whether the treatment method was different between symptomatic and asymptomatic cracked teeth. In both 2009 and 2019, symptomatic cracked teeth were more likely to be treated with RCT and prov $\rightarrow \mathrm{Cr}$. A previous study found that cracked teeth with severe sensitivity to cold were more likely to receive root canal treatment. ${ }^{14} \mathrm{~A}$ probable explanation for this finding is that symptomatic cracked teeth are more likely to have an inflamed pulp.

Unfavorable prognoses have been reported for cracked teeth with a probing depth exceeding $4 \mathrm{~mm}$, and the likelihood of requiring root canal treatment becomes higher as the probing depth increases. ${ }^{9,12,14}$ The presence of a deep periodontal pocket implies that a crack may progress more deeply into the root, undermining the support provided by the periodontium. ${ }^{5,9,24}$ The results of this study also showed that the rate of root canal treatment increased as the periodontal probing depth became deeper in both 2009 and 2019. Furthermore, it is reasonable to predict that deeper periodontal pockets are associated with more severe crack progression. Microleakage from the crack line can also increase pulp inflammation. ${ }^{5} \mathrm{~A}$ probing depth of $>6 \mathrm{~mm}$ was associated with a higher likelihood of persistent symptoms at 3 and 6 months.

It has been reported that success decreased with increasing pulpal involvement and that the success rate of cracked teeth with apical lesions was lower than that of cracked teeth without apical lesions. ${ }^{910}$ Because of variability in outcomes, extraction has been recommended for cracked teeth with necrotic pulp. ${ }^{5}$ In this study, in both 2009 and 2019, cracked teeth in which an apical lesion was observed in the initial examination showed a relatively high rate of persistent symptoms at 3-month follow-up. Thus, observing an apical lesion in the initial examination may have implications for a short-term poor prognosis, and a long-term follow-up study is needed to predict the success rate of cracked teeth with apical lesions.
Limitations of this study include the small number of patients in 2009 and the short follow-up period in 2019. Further studies involving larger samples of patients with long-term follow-up are required.

\section{Conclusion}

Through a comparison of data on cracked teeth obtained at an interval of 10 years, we found that the rate of root canal treatment was lower in 2019 to 2020 than it was 10 years ago. Furthermore, probing depth was the most important factor for determining the prognosis of cracked teeth both in 2009-2010 and in 2019-2020.

\section{Funding}

This work was supported by a National Research Foundation of Korea grant funded by the Korean government (No. NRF-2019R1A2C1005247).

\section{Conflict of Interest}

None declared.

\section{References}

1 Cameron CE. Cracked-tooth syndrome. J Am Dent Assoc 1964; 68:405-411

2 American Association of Endodontists. Endodontics: Colleagues for Excellence - Cracking the Cracked Tooth Code: Detection and Treatment of Various Longitudinal Tooth Fractures. Chicago: American Association of Endodontists; 2008

3 Türp JC, Gobetti JP. The cracked tooth syndrome: an elusive diagnosis. J Am Dent Assoc 1996;127(10):1502-1507

4 Ailor JE Jr. Managing incomplete tooth fractures. J Am Dent Assoc 2000;131(8):1168-1174

5 Berman LH, Kuttler S. Fracture necrosis: diagnosis, prognosis assessment, and treatment recommendations. J Endod 2010;36(3):442-446

6 Bergenholtz G. Pathogenic mechanisms in pulpal disease. J Endod 1990;16(2):98-101 
7 Cameron CE. The cracked tooth syndrome: additional findings. J Am Dent Assoc 1976;93(5):971-975

8 Rivera EM, Williamson A. Diagnosis and treatment planning: cracked tooth. Tex Dent J 2003;120(3):278-283

9 Tan L, Chen NN, Poon CY, Wong HB. Survival of root filled cracked teeth in a tertiary institution. Int Endod J 2006; 39(11):886-889

10 Krell KV, Caplan DJ. 12-month success of cracked teeth treated with orthograde root canal treatment. J Endod 2018; 44(4):543-548

11 Touré B, Faye B, Kane AW, Lo CM, Niang B, Boucher Y. Analysis of reasons for extraction of endodontically treated teeth: a prospective study. J Endod 2011;37(11):1512-1515

12 Kang SH, Kim BS, Kim Y. Cracked teeth: distribution, characteristics, and survival after root canal treatment. J Endod 2016; 42(4):557-562

13 Krell KV, Rivera EM. A six year evaluation of cracked teeth diagnosed with reversible pulpitis: treatment and prognosis. J Endod 2007;33(12):1405-1407

14 Kim SY, Kim SH, Cho SB, Lee GO, Yang SE. Different treatment protocols for different pulpal and periapical diagnoses of 72 cracked teeth. J Endod 2013;39(4):449-452
15 Hiatt WH. Incomplete crown-root fracture in pulpal-periodontal disease. J Periodontol 1973;44(6):369-379

16 Roh BD, Lee YE. Analysis of 154 cases of teeth with cracks. Dent Traumatol 2006;22(3):118-123

17 Seo DG, Yi YA, Shin SJ, Park JW. Analysis of factors associated with cracked teeth. J Endod 2012;38(3):288-292

18 Abbott P, Leow N. Predictable management of cracked teeth with reversible pulpitis. Aust Dent J 2009;54(4):306-315

19 Lynch CD, McConnell RJ. The cracked tooth syndrome. J Can Dent Assoc 2002;68(8):470-475

20 Ehrmann EH, Tyas MJ. Cracked tooth syndrome. Aust Dent J 1990;35(4):390-391

21 Lubisich EB, Hilton TJ, Ferracane J. Northwest Precedent. Cracked teeth: a review of the literature. J Esthet Restor Dent 2010;22(3):158-167

22 Ellis SG. Incomplete tooth fracture-proposal for a new definition. Br Dent J 2001;190(8):424-428

23 Kahler $\mathrm{W}$. The cracked tooth conundrum: terminology, classification, diagnosis, and management. Am J Dent 2008;21(5): 275-282

24 Gutmann JL, Rakusin H. Endodontic and restorative management of incompletely fractured molar teeth. Int Endod J 1994;27(6):343-348 